4. In Anbetracht der schon im ersten Vorwort betonten Bedeutung anschaulicher Abbildungen für das Verständnis wissenschaftlicher Fragen wurde ihre Anzahl weiter vermehrt. So kamen neu hinzu die Abbildungen 30 (Zustandekommen des osmotischen Drucks), 86 (Räumliche Molekularformel des weißen Phosphors $\mathrm{P}_{4}$ ), 87 (Gitterstruktur des schwarzen Phosphors $\mathrm{P}_{\infty}$ ), 89 (Räumliche Molekularformel des Phosphortrioxyds $\left.\left(\mathrm{P}_{2} \mathrm{O}_{3}\right)_{2}\right), 92$ (Gitterstruktur des metallischen Arsens $\mathrm{As}_{\infty}$ ), 97 (Molekularstruktur des Kohlenstoffmonofluorids $\left.(\mathrm{CF})_{\infty}\right), 114$ (Schema des Bleiakkumulators), 158 (Wirkungsweise des Cyclotrons), 161 (Schema der Uranspaltung), 162 (Ausbeuten der Uranspaltung), 163 (Uran-Pile), 164 (Schema der gesteuerten Kern-Kettenreaktion), 165 (Schema der ungesteuerten Kern-Kettenreaktion).

Trotz dieser umfangreichen Vermehrung des Wissensstoffes, die auch in der Erweiterung des Registers um mehrere tausend Stichworte und in der starken Vermehrung der Zahl der Seitenhinweise innerhalb des Textes zum Ausdruck kommt, konnte durch Streichung entbehrlicher und überholter Abschnitte der Zuwachs des äußeren Umfangs auf 56 Seiten (42 Seiten Text, 14 Seiten Register) begrenzt werden.

München, im Januar 1951.

Egon Wiberg

\title{
Aus dem Vorwort zur 28. und 29. Auflage
}

Die starke Nachfrage nach dem vorliegenden Lehrbuch machte schon kurz nach Erscheinen der letzten Doppelauflage die Vorbereitung einer neuen Doppelauflage erforderlich. Trotz der Kürze der für die Ưberarbeitung zur Verfügung stehenden Zeit wurden auch dieses Mal zahlreiche Ergänzungen und Umänderungen vorgenommen. Sie betreffen namentlich das Gebiet der Hydride und Mischhydride, auf dem in letzter Zeit zahlreiche Fortschritte erzielt werden konnten. So wurden u. a. neu aufgenommen : Der Beryllium - aluminium - wasserstoff $\mathrm{BeH}_{2} \cdot 2 \mathrm{AlH}_{3}$ (S. 402), der Magnesium wasserstoff $\mathrm{MgH}_{2}$ und seine Mischhydride mit Bor- und Aluminiumwasserstoff, $\mathrm{MgH}_{2} \cdot 2 \mathrm{BH}_{3}$ bzw. $\mathrm{MgH}_{2} \cdot 2 \mathrm{AlH}_{3}$ (S. 404f.), der Gallium-aluminium-wasserstoff $\mathrm{GaH}_{3} \cdot 3 \mathrm{AlH}_{3}$ (S. 398), der Indium-aluminium-wasserstoff $\mathrm{InH}_{3} \cdot 3 \mathrm{AlH}_{3}$ (S. 398), der Thallium-gallium-wasserstoff $\mathrm{TlH}_{3} \cdot 3 \mathrm{GaH}_{3}$ (S. 399), der Zinnaluminium-wasserstoff $\mathrm{SnH}_{4} \cdot 4 \mathrm{AlH}_{3}$ (S. 355) und der Titan-aluminiumwasserst off $\mathrm{TiH}_{4} \cdot 4 \mathrm{AlH}_{3}$ (S. 502). Modernisiert und erweitert wurden die Abschnitte über die Darstellung von Borwasserstoffen (S. 368), Siliciumwasserstoffen (S. 319), Germaniumwasserstoffen (S. 350) und Zinkwasserstoff (S. 475), nachdem in der Einwirkung von Lithium-aluminium-wasserstoff $\operatorname{LiAlH}_{4}$ (S.388) auf Metallchloride eine neue, vorteilhafte Methode zur Darstellung von Hydriden und Mischhydriden vorliegt. Eine neue Fassung erhielten die Abschnitte über das Sch wefel monoxyd (S. 201), die Disproportionierung und Zersetzung des Hydroxylamins (S. 244 f.) und die Enthärtung des Wassers (S.410). Die Actinidennatur des Urans wurde ausführlicher begründet (S. 520). Darüber hinaus finden sich an zahlreichen anderen Stellen Erweiterungen und Ergänzungen, wie etwa über die 
natürliche Radioaktivität des Indiums (S. 569), die Elementumwandlung mit Kohlenstoffkernen (S. 581, 606), die Radioaktivität des Neutrons (S. 585), die Periodizität der Actiniden (S. 606) oder Ausnahmen der Matradchschen Regel (S. 558). Weiterhin wurde der Text sorgfältig auf Druckfehler und mißverständliche Formulierungen geprüft und an manchen Stellen (z. B. bezüglich der Anregung von Freiheitsgraden der Rotation, S. 78 f.) berichtigt.

München, im Oktober 1951.

Egon Wiberg

\section{Aus dem Vorwort zur 32. und 33. Auflage}

Auch die vorliegende neue Auflage ist an vielen Stellen verbessert und erweitert worden, soweit dies ohne Umbruch des gesamten Satzes möglich war. So wurde, um nur einiges herauszugreifen, die Ableitung des osmotischen Drucks (S. 55) und der Dampfdruckerniedrigung (S. 57) abgeändert, der Begriff der „Ladungszahl“ (S. 148) und „Bindungszahl“ (S. 150) eingeführt, eine Reihe von physikalischen Daten (Schmelzpunkte, Siedepunkte, Halbwertszeiten usw.) nachgetragen oder korrigiert (z. B. S. $220,562,564,602 / 603$ ), die relative thermodynamische Beständigkeit der Phosphormodifikationen richtiggestellt (S. 253, 254), der Begriff der Isosterie durch zusätzliche Beispiele erläutert (S. 372), die Chemie des Boroxols und seiner Derivate durch weitere Angaben bereichert (S. 375/76, Anm. 3), der Abschnitt über Aluminiumwasserstoff vervollständigt (S. $387 \mathrm{f}$.), die Trennung der Lantha. niden durch Ionenaustauscher behandelt (S. 488f.), der Abschnitt über Technetium erweitert (S. 597 f.), die Tabelle der Normal- und Umladungspotentiale des Urans, Neptuniums und Plutoniums auf den neuesten Stand gebracht und durch die entsprechenden Werte des A mericiums ergänzt (S. 604). Darüber hinaus wurden, wo erforderlich, Druckfehler beseitigt, unklare Textstellen präzisiert oder veranschaulicht, experimentell nicht genügend gestützte Auffassungen deutlicher als solche gekennzeichnet, die Nomenklatur weiter dem internationalen Sprachgebrauch angepaßt und zahlreiche sonstige Einfügungen vorgenommen, wie schon die Erweiterung des Registers um einige zwanzig Stichworte zeigt.

München, im September 1953.

Egon Wiberg 